\title{
Complexity of a quasiperiodically driven spin system
}

\author{
Arkady S Pikovsky, Michael A Zaks and Jürgen Kurths \\ Max-Planck-Arbeitsgruppe 'Nichtlineare Dynamik', Universität Potsdam, Potsdam, Germany
}

Received 16 August 1995

\begin{abstract}
A quasiperiodically forced purely quantum spin- $\frac{1}{2}$ system can exhibit rather irregular behaviour with a continuous power spectrum. We have shown analytically and numerically, that in these regimes both the topological entropy of a corresponding symbolic sequence and the maximal Lyapunov exponent vanish.
\end{abstract}

\section{Introduction}

The question of whether chaotic motion is possible in quantum systems has attracted wide interest recently. The answer depends on what the definition of chaos is. In classical mechanics one usually defines chaotic motion as irregular motion which depends sensitively on initial conditions [1]. Irregularity means that the motion is not regularly recurrent, e.g. it has decaying correlations and a broad-band component in the power spectrum. Sensitivity to initial conditions is measured quantitatively by the largest Lyapunov exponent, which should be positive for chaos. An extension of this notion from classical to quantum systems is rather non-trivial [2-5]. On one hand, in quantum systems the Lyapunov exponent is not positive, and therefore there is no sensitive dependence on initial conditions (the most visual manifestation of this is the reversibility of quantum dynamics [6]). On the other, a rather irregular motion with a continuous spectrum can be observed in quantum systems [7-9]. The latter case deserves detailed investigation. Such behaviour can also occur in classical systems, but there it can be considered as a rather unusual situation at the border between order and chaos [10, 11]. In quantum systems, where there is no chaos in the classical sense, such regimes may be the most complex possible. The goal of this paper is to investigate the complexity of quantum systems having a continuous spectrum.

The model which we study here is a quasiperiodically forced quantum system. First, it should be noted that a quasiperiodic force has minimal complexity for a driven quantum system to demonstrate non-trivial behaviour (in the case of a periodic driving force the response can only be quasiperiodic, as follows from the Floquet theorem). Our particular model is a driven spin- $\frac{1}{2}$ system. A number of numerical and analytical studies were devoted to this problem. Pomeau et al [12] studied numerically the quasiperiodically forced twolevel system, and observed correlations and spectra typical of chaotic behaviour. More thorough numerical studies [13, 8] showed that the conclusions of [12] could be caused by insufficient numerics. Further studies of the correlation properties of this system [7-9, 14-17] demonstrated that depending on the form of the force, the response of the spin- $\frac{1}{2}$ system can have a discrete, singular continuous, or absolutely continuous spectrum. Quite recently, the complexity of the dynamics of this system was investigated by Crisanti et al [18]; they suggested that its topological entropy is positive. More precisely, they have 
introduced symbolic dynamics, and provided numerical results showing that the number of possible words grows exponentially with the word length.

In this paper we address the problem of complexity of the regimes having a continuous spectrum. We show analytically and numerically that the symbolic dynamics of the quasiperiodically driven spin- $\frac{1}{2}$ system is rather simple: the number of possible words grows as a power of their length, even when the system has a continuous (singular or absolute) spectrum. We also discuss the Lyapunov exponent for this system and explain the results of [19], where the exponential sensitivity for this system was reported.

\section{The model}

The spin- $\frac{1}{2}$ system in a time-dependent magnetic field $S(t)$ is described by the Hamiltonian

$$
H(t)=\frac{1}{2} \omega \sigma_{z}+\frac{1}{2} S(t) \sigma_{x}
$$

where $\sigma_{x}$ and $\sigma_{z}$ are the Pauli matrices

$$
\sigma_{x}=\left(\begin{array}{cc}
0 & 1 \\
1 & 0
\end{array}\right) \quad \sigma_{z}=\left(\begin{array}{cc}
1 & 0 \\
0 & -1
\end{array}\right) .
$$

The Schrödinger equation for a spinor $\left(\psi_{1}, \psi_{2}\right)$ is (we take $\hbar=1$ )

$$
\begin{aligned}
& \mathrm{i} \frac{\mathrm{d} \psi_{1}}{\mathrm{~d} t}=-\frac{1}{2} \omega \psi_{1}+\frac{1}{2} S(t) \psi_{2} \\
& \mathrm{i} \frac{\mathrm{d} \psi_{2}}{\mathrm{~d} t}=\frac{1}{2} \omega \psi_{2}+\frac{1}{2} S(t) \psi_{1} .
\end{aligned}
$$

We rewrite this system in terms of the observable Bloch variables

$$
A=\left|\psi_{2}\right|^{2}-\left|\psi_{1}\right|^{2} \quad B=\mathrm{i}\left(\psi_{2} \psi_{1}^{*}-\psi_{1} \psi_{2}^{*}\right) \quad C=\psi_{2} \psi_{1}^{*}+\psi_{1} \psi_{2}^{*}
$$

as

$$
\begin{aligned}
& \frac{\mathrm{d} A}{\mathrm{~d} t}=S(t) B \\
& \frac{\mathrm{d} B}{\mathrm{~d} t}=-S(t) A-\omega C \\
& \frac{\mathrm{d} C}{\mathrm{~d} t}=\omega B .
\end{aligned}
$$

Now suppose that the driving magnetic field $S(t)$ is a sequence of $\delta$-kicks with the basic period $T$ and time-dependent amplitude $R$ :

$$
S(t)=\sum_{n=-\infty}^{\infty} R_{n} \delta(t-n T) .
$$

Then between the kicks equations (2) describe the free rotation of the spin:

$$
\begin{aligned}
& \tilde{A}=A \\
& \tilde{B}=\cos (\omega T) B-\sin (\omega T) C \\
& \tilde{C}=\sin (\omega T) B+\cos (\omega T) C
\end{aligned}
$$

and during the kick only $A$ and $B$ vary, hence

$$
\begin{aligned}
\bar{A} & =\cos (R) \tilde{A}+\sin (R) \tilde{B} \\
\bar{B} & =-\sin (R) \tilde{A}+\cos (R) \tilde{B} \\
\bar{C} & =\tilde{C} .
\end{aligned}
$$


The combination of the two rotations (4) and (5) gives the linear mapping

$$
\left(\begin{array}{l}
A_{n+1} \\
B_{n+1} \\
C_{n+1}
\end{array}\right)=\left(\begin{array}{ccc}
\cos \left(R_{n}\right) & \sin \left(R_{n}\right) \cos (\omega T) & -\sin \left(R_{n}\right) \sin (\omega T) \\
-\sin \left(R_{n}\right) & \cos \left(R_{n}\right) \cos (\omega T) & -\cos \left(R_{n}\right) \sin (\omega T) \\
0 & \sin (\omega T) & \cos (\omega T)
\end{array}\right)\left(\begin{array}{c}
A_{n} \\
B_{n} \\
C_{n}
\end{array}\right)
$$

which evidently conserves the integral

$$
A^{2}+B^{2}+C^{2}=1 \text {. }
$$

We will further assume that the quasiperiodic sequence $R_{n}$ is generated by the following dynamical system:

$$
\begin{array}{lc}
\phi_{n+1}=\phi_{n}+\Omega & \bmod 1 \\
R_{n}=\kappa \Phi\left(\phi_{n}\right) & \Phi(\phi+1)=\Phi(\phi) .
\end{array}
$$

An irrational $\Omega$ in the circle map (8) produces a quasiperiodic sequence $\phi_{n}$, and the amplitudes $R_{n}$ are defined via the modulation function $\Phi(\phi)$.

\section{Correlation properties}

As has been shown in $[9,17]$, the correlation properties of the observables $A, B, C$ depend on the form of the modulation function $\Phi(\phi)$. If this function is continuous, e.g.

$$
\Phi(\phi)=\sin (2 \pi \phi)
$$

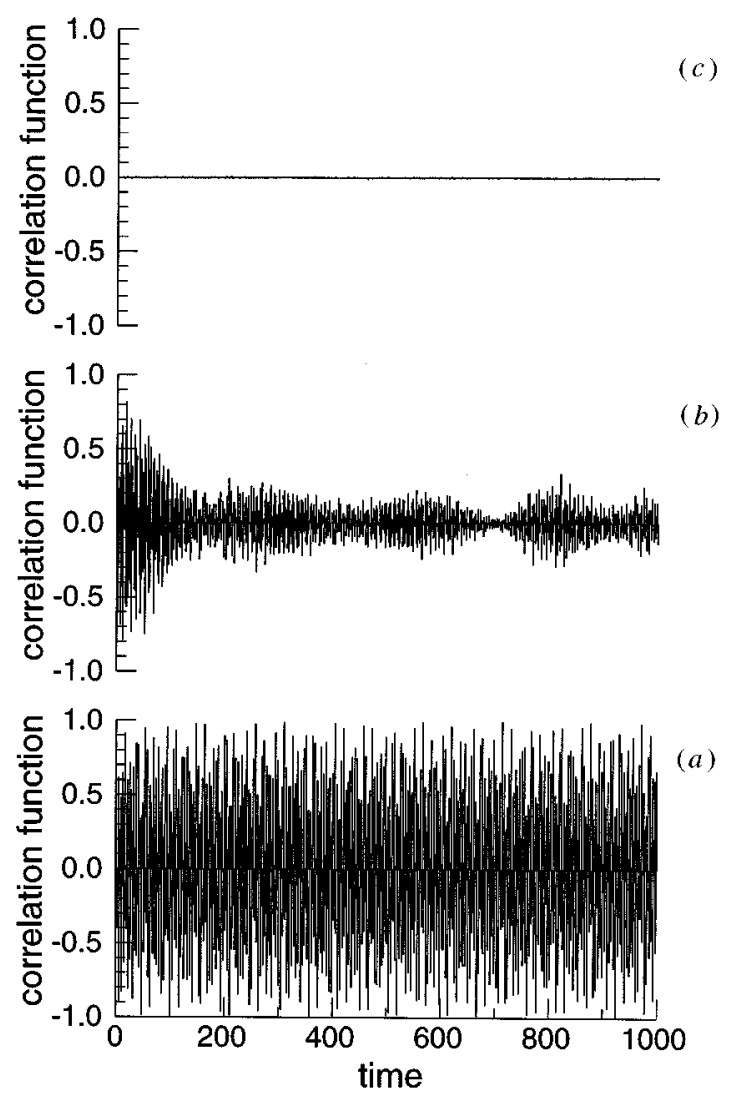

Figure 1. The autocorrelation function of the observable $B_{n}$ for the three types of modulation function $\Phi$ : $(a)$ modulation function (10) gives a discrete spectrum [16]; $(b)$ modulation function (11) gives a singular continuous spectrum (see [9] for more details); (c) modulation function (12) gives an absolutely continuous spectrum. In cases $(a)$ and $(b)$ the parameters of the system are $\kappa=\pi / 4, \beta=\frac{1}{2}, \omega=\xi^{-1}$, and $\Omega=\xi^{-2}$, where $\xi$ is the real root of the equation $\xi^{3}-\xi-1$. In $(c) \Omega=(\sqrt{5}-1) / 2$. 
the observables vary in time quasiperiodically [16]. A discontinuity in $\Phi(\phi)$ destroys the regularity of the dynamics, and the spectrum of the dynamical variables $A, B, C$ is continuous. In $[9,17]$ it has been shown that in the case of a piecewise-constant modulation function

$$
\Phi(\phi)=\left\{\begin{array}{lll}
1 & \text { if } \quad 0 \leqslant \phi<\beta \\
-1 & \text { if } \quad \beta \leqslant \phi<1
\end{array}\right.
$$

the spectrum is singular continuous (fractal). These conclusions are based mainly on the numerics, however, they are supported by a renormalization-group analysis and by a rigorous proof for a particular choice of parameters [9].

Another choice of the modulation function

$$
\Phi(\phi)=\phi
$$

with $\kappa=1$, and $\omega T=2 \pi n$ gives an absolutely continuous spectrum [8]. We illustrate this with calculations of the autocorrelation function in figure 1 (remember that the power spectrum is the Fourier transform of the autocorrelation function).

\section{Symbolic dynamics}

\subsection{Definition}

As follows from (6) and (7), a state $A, B, C$ can be considered as a point on the unit sphere, and the dynamics are the rotations of this sphere. In [18] it was suggested to describe a point on the sphere with two symbols $[0,1]$, by simply dividing the sphere into two parts by the equator. In this way, a trajectory is represented by a binary string, and the question is how to describe the complexity of this string. There are many complexity measures (see, for example, [20]), but we will use the one that can be calculated in a rather simple way [21]. It is defined via the complexity function $N(n)$, which is equal to the number of all different words of length $n$ in the sequence. For periodic sequences, $N$ eventually does not grow with $n$, while for simple quasiperiodic sequences (like the one generated by the mappings (8), (11)) one has $N_{q p}(n) \sim$ constant $\times n$. If $N(n)$ grows with $n$ exponentially, the exponent is the topological entropy:

$$
h_{\text {top }}=\lim _{n \rightarrow \infty} \frac{1}{n} \log _{2} N(n) \text {. }
$$

Chaotic systems have positive topological entropy; their symbolic sequences are nondistinguishable from random ones [22]. Below we analytically and numerically estimate the complexity of the symbolic sequence generated by the quasiperiodically driven spin- $\frac{1}{2}$ system.

\subsection{Analytical results}

Consider the quasiperiodically forced spin- $\frac{1}{2}$ system (6) with the modulation function (11). This system is similar to that investigated in [18]. Note first, that in this case the external force (given by equations (8), (9), (11)) is represented by a quasiperiodic binary sequence. The dynamics of the quantum system itself consists of the rotations of the sphere. Let us look at how many different symbolic sequences can be maximally generated by such a dynamics, for a given sequence of external force. If the external force is fixed, different symbolic sequences are produced by different initial conditions, and we want to calculate the number of these. Our partition of the sphere is given by the equator (great circle) $S$. 
The transformation (6) is a rotation, which is invertible. Therefore, we can define preimages of the equator under this transformation $S_{-1}, S_{-2}$, etc. Clearly, all these preimages are also great circles (circles with radius equal to the radius of the sphere) on the sphere, and they generate a fine partition of the sphere into domains with the same symbolic sequences [23]. Thus, we have to calculate, into how many parts $K(n)$ can the sphere be divided by $n$ great circles. In general, each new great circle on the sphere intersects each of previous circles twice, and each such intersection produces a new domain. Thus, we have the recursive relation

$$
K(n)=K(n-1)+2(n-1) .
$$

Taking into account the obvious initial condition $K(1)=2$, we get

$$
K(n)=n^{2}-n+2 .
$$

The quantity $K(n)$ measures the number of possible symbolic sequences of length $n$ for the driven spin- $-\frac{1}{2}$ system, for the given sequence of the external force. To estimate the total number of possible symbolic sequences we have to multiply $K(n)$ with the number of possible sequences of the external force. Because the external force is quasiperiodic, the latter number $N_{q p}(n)$ can be estimated as $N_{q p}(n)<C_{1} n$. Therefore, the final estimate for the complexity function $N(n)$ is

$$
N(n)<C_{1} n\left(n^{2}-n+2\right) .
$$

Evidently, the topological entropy of this complexity function is zero.

Similar arguments can be applied to the case of the modulation function (12), when the spectrum is absolutely continuous. Because $\omega T=2 \pi n$, the rotation (4) is the identity, and the observable $C$ is constant. So we can introduce the phase $\theta$ as $(A, B)=$ $\sqrt{1-C^{2}}(\cos (2 \pi \theta), \sin (2 \pi \theta))$, and write the mapping as

$$
\begin{aligned}
& \phi_{n+1}=\phi_{n}+\Omega \quad \bmod 1 \\
& \theta_{n+1}=\theta_{n}+\phi_{n} \quad \bmod 1 .
\end{aligned}
$$

This system is known in the ergodic theory as the skew product [24]. It is the mapping of the torus $0 \leqslant \phi<1,0 \leqslant \theta<1$ and its complexity can be calculated as follows. As the basic partition we can choose, similar to [18], the domains $0 \leqslant \theta<\frac{1}{2}$ and $\frac{1}{2} \leqslant \theta<1$ (this choice means that the states with positive and negative observables $B$ are marked by different symbols). These domains are separated by the lines $S_{0}^{1}: \theta=\frac{1}{2}, S_{0}^{2}: \theta=0$. The $n$th preimages of these lines are, according to (16), (17), $S_{n}^{1}: \theta=\frac{1}{2}-n(\phi+\Omega)$, $S_{n}^{2}: \theta=-n(\phi+\Omega)$. The number of intersections of the lines $S_{n}^{1,2}$ and $S_{m}^{1,2}$ is $|n-m|$. At each intersection of $S_{n}^{1,2}$ with previous $S_{k}^{1,2}, k<n$, at most one new domain appears. Therefore we can estimate the number of domains as

$$
N(n) \leqslant N(n-1)+2\left(n^{2}+n\right)
$$

which yields for the complexity function

$$
N(n)<C_{2} n^{3} .
$$

Again, the topological entropy of this sequence is zero, although it is completely uncorrelated. 


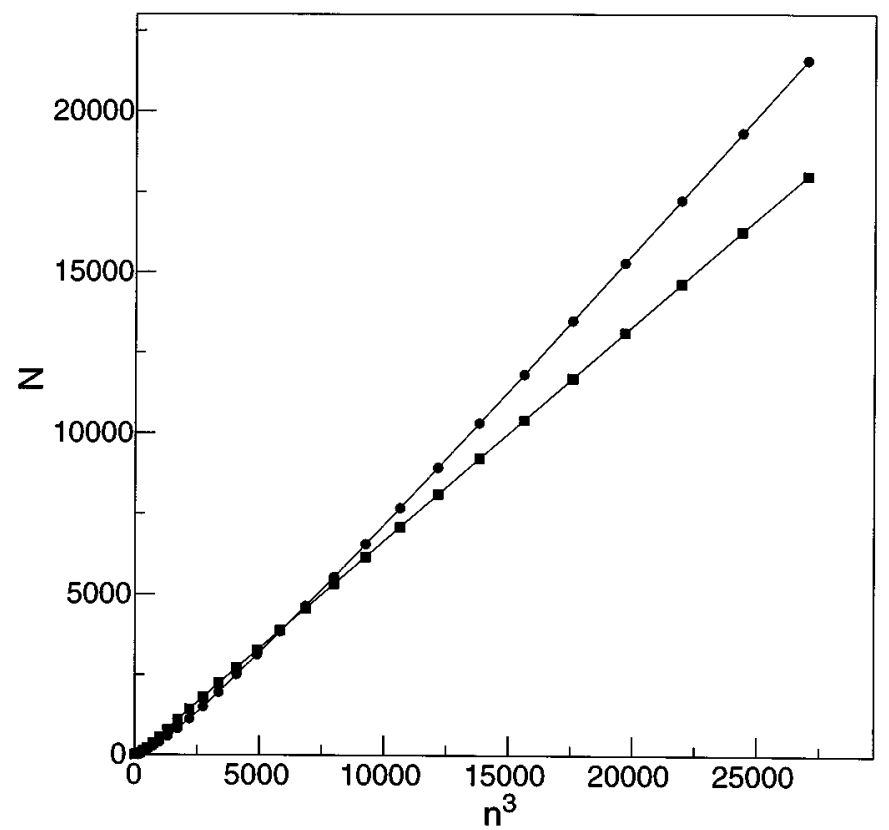

Figure 2. The number of possible words $N$ versus $n^{3}$ for cases $(b)$ and $(c)$ of figure 1 (circles and squares, respectively).

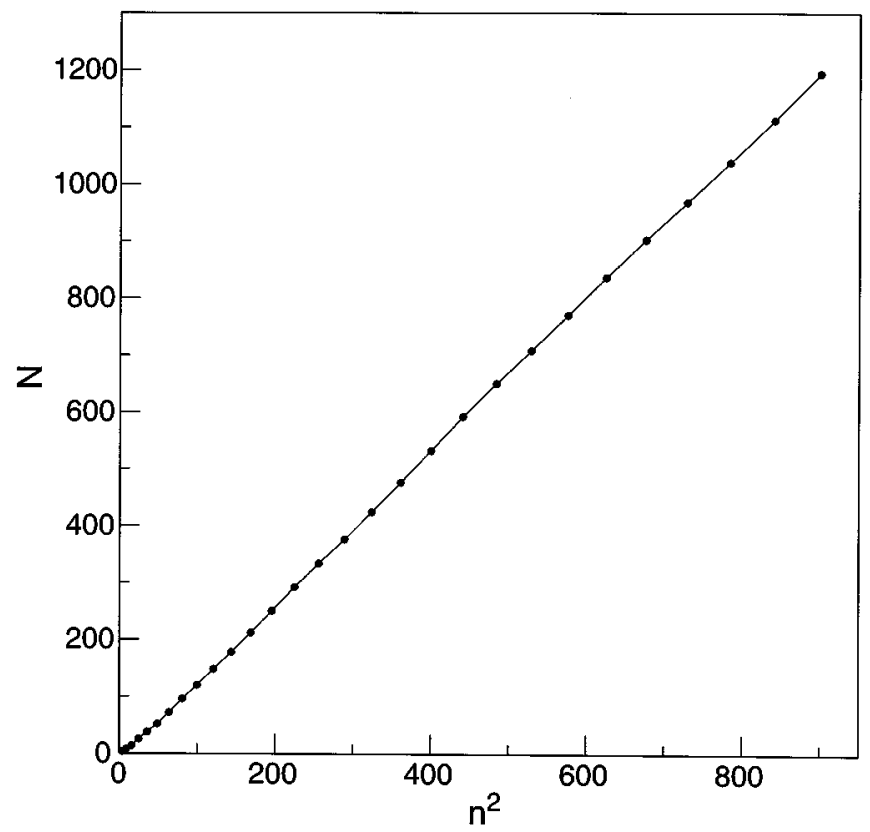

Figure 3. The number of possible words $N$ versus $n^{2}$ for case (a) of figure 1 .

\subsection{Numerical results}

We have checked the formulae (15) and (19) numerically, using straightforward iterations of the mapping (6). The results are presented in figures 2 and 3. One can see, that in 
all cases the complexity $N(n)$ grows as a power law. For the quasiperiodic motion, we have obtained $N(n) \sim n^{2}$, while for the behaviour with singular continuous and absolutely continuous spectrum we have $N(n) \sim n^{3}$ in accordance with the analytical results (15) and (19). The numerical data indicate clearly that the topological entropy of the quasiperiodically driven spin- $\frac{1}{2}$ system is zero.

\section{Exponential sensitivity}

Recently, Blümel [19] considered a quasiperiodically forced spin- $\frac{1}{2}$ system very similar to that described in section 2, and claimed that it has a positive Lyapunov exponent and a very strong form of chaos. This result contradicts the common belief that quantum systems have no positive Lyapunov exponents, and is, therefore, worth discussing (see also [25]).

In our notation, the system considered in [19] is the model (6), (8), (9), (11), with the parameters $\omega T=2 \pi n, \beta=\Omega=(\sqrt{5}-1) / 2$. This choice of the frequency $\Omega$ as the reciprocal of the golden mean produces the Fibonacci sequence as the external force. The characteristic times for this sequence are the Fibonacci numbers $F_{k}$ obeying the recurrence $F_{k+1}=F_{k}+F_{k-1}, F_{1}=F_{2}=1$. The dynamics of the spin- $\frac{1}{2}$ system consist of simple rotations (5), and can be described by a phase $\theta$, which also obeys the recurrence

$$
\theta_{k+1}=\theta_{k}+\theta_{k-1} \text {. }
$$

If equation (20) is considered as a dynamical system, its Lyapunov exponent is positive: $\lambda=\log [(\sqrt{5}+1) / 2]$, and the conclusions of [19] are based on this observation.

To explain the contradiction, it is sufficient to note that $k$ is not the real time, but a renormalized one. The phase $\theta_{k}$ corresponds to the real time $n=F_{k}$, which grows with $k$ with the same exponent $\lambda=(\sqrt{5}+1) / 2$. Thus, perturbations of the phase grow with real time $n$ linearly:

$$
\Delta(\theta) \sim \mathrm{e}^{\lambda k} \approx F_{k}=n .
$$

The linear growth of perturbations is typical for quasiperiodic systems, but as we have shown above, may also occur in systems with continuous spectrum.

The system considered in [19] is, therefore, not chaotic — the conclusions of [19] are based on the muddle of the real and the renormalized time.

\section{Conclusion}

We have considered the purely quantum spin- $\frac{1}{2}$ dynamics driven by a quasiperiodic external field. The dynamics of this system, albeit being not chaotic in the sense of classical dynamical systems, can be rather irregular. In particular, depending on the form of the external force, observables can have a discrete, singular continuous, or absolutely continuous power spectrum. We have shown that in all these cases the complexity function allows one to distinguish the dynamics from a chaotic one: the topological entropy of the observed sequence of symbols is zero. This system also has a vanishing maximal Lyapunov exponent.

\section{Acknowledgments}

We thank P Grassberger, G Mantica, A Politi, D Shepelyansky, U Smilansky, A Vulpiani, and A Witt for useful discussions and I Guarneri for sending his paper [17] prior to publication. MZ acknowledges support from the Max-Planck-Gesellschaft. 


\section{References}

[1] Lichtenberg A J and Lieberman M A 1992 Regular and Chaotic Dynamics (New York: Springer)

[2] Chirikov B, Izrailev F and Shepelyansky D 1981 Sov. Sci. Rev. C 2209

[3] Casati G (ed) 1985 Chaotic Behavior in Quantum Systems (New York: Plenum)

[4] Haake F 1990 Quantum Signatures of Chaos (Berlin: Springer)

[5] Reichl L E 1992 The Transition to Chaos in Conservative Classical Systems: Quantum Manifestations (New York: Springer)

[6] Casati G, Chirikov B, Guarneri I and Shepelyansky D 1987 Phys. Rep. 15477

[7] Luck J M, Orland H, and milansky U S 1988 J. Statist. Phys. 53551

[8] Blekher P M, Jauslin H R, and Lebowitz J L 1992 J. Statist. Phys. 68271

[9] Feudel U, Pikovsky A S and Zaks M A 1995 Phys. Rev. E 511762

[10] Broer H and Takens F 1993 Arch. Rational Mech. Anal. 12413

[11] Pikovsky A S, Zaks M A, Feudel U and Kurths J 1995 Phys. Rev. E 52285

[12] Pomeau Y, Dorizzi B and Grammaticos B 1986 Phys. Rev. Lett. 56681

[13] Badii R and Meier P F 1987 Phys. Rev. Lett. 581045

[14] Sutherland B 1986 Phys. Rev. Lett. 57770

[15] Graham R 1989 Europhys. Lett. 8717

[16] Geisel T 1990 Phys. Rev. A 412989

[17] Guarneri I and Meo M 1995 J. Phys. A: Math. Gen. 282717

[18] Crisanti A et al 1994 Phys. Rev. E 50138

[19] Blümel R 1994 Phys. Rev. Lett. 73428

[20] Wackenbauer R et al 1994 Chaos, Solitons and Fractals 4133

[21] Queffélec M 1987 Substitution Dynamical Systems-Spectral Analysis (Springer Lecture Notes in Mathematics 1294) (Berlin: Springer)

[22] Alekseev V M and Yakobson M V 1981 Phys. Rep. 75287

[23] Martin N F G and England J W 1981 Mathematical Theory of Entropy (Reading, MA: Addison-Wesley)

[24] Cornfeld I P, Fomin S V and Sinai Ya G 1982 Ergodic Theory (New York: Springer)

[25] Schack R 1995 Phys. Rev. Lett. 75581 